\title{
Prevalence and antibiotic Susceptibility of Bacterial Strains Isolated From Urinary Tract Infections in Warangal, India.
}

\author{
Adothu Ramakrishna ${ }^{1}$, M.A.Singaracharya ${ }^{2}$ \\ Department of Microbiology Kakatiya UniversityWarangal, India ${ }^{l}$. \\ Department of Microbiology, Kakatiya University, Warangal, India ${ }^{2}$.
}

\begin{abstract}
Urinary tract infection (UTI) is one of the most common bacterial diseases worldwide that can be presented asymptomatic or symptomatic, characterized by a wide range of symptoms from mild irritative voiding to bacteremia, sepsis, or even death. In latter decades, widespread utilization of antibiotics has resulted in increased incidence of antibiotic resistance among urinary tract pathogens all over the world. The aim of this study was to determine patterns of antibiotic resistance bacteria isolated from urinary tract infection $s$ in the city of Warangal. This descriptive study was conducted in the city of Warangal. Bacterial isolates were identified by standard biochemicals tests. Antibacterial susceptibility test was performed by Kirby-Bauer method against some common antibiotics for gram negative and gram positive bacteria. In this study, 230 (8.06\%) out of 2852 patients showed positive urine cultures of which there were 204 (88.69\%) females and 26 (11.3\%) males, 180 (83.17\%) cases of isolated bacteria were gram negative bacilli while 50 (21.73\%) cases were gram positive cocci. E.coli isolates, as the most frequent gram negative bacteria, showed high resistance to cephalothin (88.16\%) and high susceptibility to nitrofurantoin (90.78\%). Also Staphylococcus saprophyticus isolates, as the most frequent gram positive bacteria, exhibited high resistance to amplicillin, tetracycline, and erythromycin (92.31\%) and high susceptibility to nitrofurantoin and vancomycin (92.3\%).Considering the results of this study, E coli isolates were the predominant pathogens in UTI. Our data demonstrated that antibiotics with the highest activity included ciprofloxacin and nitrofurantoin and those with the least activity included ampicillin and Sulfamethoxazole-trimethoprim. Our findings reinforce the need for ongoing investigations to show the trends of antibiotic resistance which can help antibiotic prescription in the clinics.
\end{abstract}

Keywords: Antimicrobial agents , Bacteremia, Gram negative bacteria ,Multidrug resistant, Urinary tract infections,

\section{Introduction}

Urinary tract infections (UTIs) are among the most common bacterial diseases worldwide (1), and the commonest genitourinary disease in children (2). UTI can be asymptomatic or symptomatic, characterized by a wide spectrum of symptoms ranging from mild irritative voiding to bacteremia, sepsis, or even death (3). UTIs account for approximately 11.3 million office visits with an overall cost of 1.6 billion dollars annually in the US health budget (4). Infection may occur at any part of the genitourinary tract, including urethra, bladder, ureter, renal pelvis, or renal parenchyma $(5,6)$. Most infections are caused by retrograde ascent of bacteria from fecal flora to bladder and kidney via urethra, especially in females whom the urethra is shorter and wider (7).

Anatomical structure of female urethra and vagina make them susceptible to trauma during sexual intercourse and bacterial passage through urethra up to the bladder during pregnancy and delivery $(8,9)$. The infection may involve only lower or both upper and lower parts of urinary tract. The term "cystitis" has been used to describe a syndrome including dysuria, frequency, and occasionally supra pubic tenderness. "Acute pyelonephritis" describes a clinical syndrome characterized by flank pain or tenderness, or both, and fever, often accompanied by dysuria, urgency, and frequency (10). Majority of UTIs are not life threatening and do not cause any irreversible damage. However, when the kidneys are involved, there is a risk of irreparable tissue damage with an increased risk of bacteremia (11).

Bacteria are the major causative organism and are responsible for more than $95 \%$ of UTI cases (12). Escherichia coli is the most frequent infecting organism in acute infections $(13,14)$. Area-specific monitoring studies aimed to understanding the types of pathogens responsible for UTIs and their resistance patterns may help clinicians to select proper empirical treatment (15).

\section{Objectives}

The aims of this study were to investigate microbial species isolated from patients with UTI and evaluate their in vitro susceptibility patterns to commonly used antimicrobial agents in Warangal, India. 


\subsection{Specimen Collection and Bacterial Isolates}

\section{Materials And Methods}

This study was conducted from November 2011 to August 2012 on 2852 untreated patients with clinical symptoms of UTI referred to a central diagnostic lab in Warangal, India. Clean catch midstream urine samples (MSU) were collected in sterile disposable containers $(4-5 \mathrm{ml})$ and transported immediately to the laboratory. Urine specimens were subjected to general urine examinations using direct microscopy for white blood cell (WBC) counting. Urine samples were cultured on 5\% blood agar, MacConkey, and Eosin-Methylene blue (EMB) agar (Hi media Ltd, Mumbai) using calibrated loops for semi-quantitative method (16) and incubated in both aerobic and anaerobic conditions for 24 hours at $37^{\circ} \mathrm{C}$. Cultures without any colony at the end of $24 \mathrm{~h}$ incubation were further incubated for $48 \mathrm{~h}$. Samples with colony count equal or more than $10{ }^{5} \mathrm{Cfu} / \mathrm{ml}$ were considered positive. The isolates were identified and confirmed using standard microbiological methods including Gram staining, colonial morphology on media, growth on selective media, lactose and mannitol fermentation, $\mathrm{H}_{2} \mathrm{~S}$ production, catalase, oxidase, coagulase, indole, and citrate utilization, and urease test.

\subsection{Antimicrobial Susceptibility Testing}

Antimicrobial susceptibility testing was performed on Mueller-Hinton agar (Hi media, Mumbai) using disk diffusion (Kirby Bauer's) technique according to Clinical and Laboratory Standards Institute (CLSI)

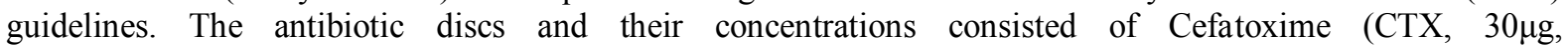

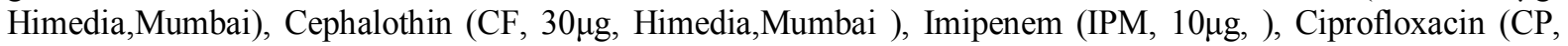

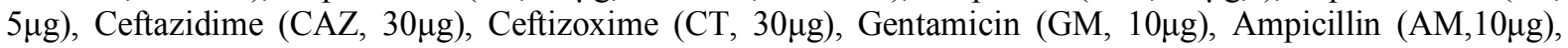

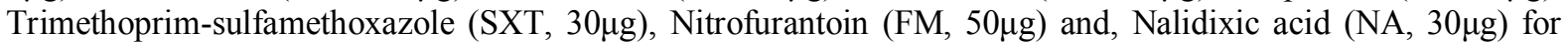

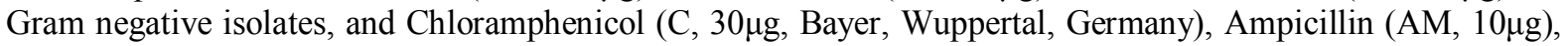

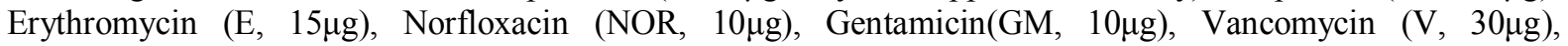

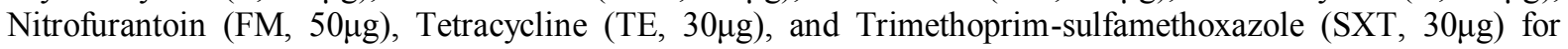
Gram positive isolates all obtained from Hi media, India.

E. coli (ATCC 25922), Staphylococcus aureus (ATCC25923), and Pseudomonas aeruginosa (ATTC 27853) were used as quality control strains.

Data were analyzed using SPSS version 12.0.1. Discrete variables were expressed as percentages and proportions were compared using the Chi-square test. Statistical significant difference was considered at value of $\mathrm{P} \leq 0.05$.

\section{Results}

In this study, $230(8.06 \%)$ out of 2852 patients showed positive urine cultures of which there were 204 (88.69\%) females and $26(11.3 \%)$ males. $180(83.17 \%)$ cases of the isolated bacteria were Gram negative bacilli while $50(21.73 \%)$ cases were Gram positivecocci. The frequency of isolated microorganisms and their relations to sex is given in Table 1.

Patients' age ranged from 10 days to 90 years with an average age of 26.17 years.

The age distribution was $22.6 \%$ for patients aged $\leq 2$ year, $9.56 \%$ for 2 to 14 years, $57.39 \%$ for 15 to 60 years, and $9.56 \%$ for $\geq 60$ years. E. coli was the most common microorganism isolated in all age-groups and both sexes. No significant difference was found between isolated bacteria and age of the patients. The isolation percentages of bacterial species were found as E. coli (66.08\%); S. saprophyticus (11.3\%); Klebsiella spp. (9.6\%); S. group B (4.34\%); Enterococcus spp. (4.34\%); S. aureus (1.7\%); Proteus spp. (1.7\%), and Enterobacter spp. $(0.86 \%)$. E. coli isolates, the predominant cause of UTIs, showed high resistance to gentamicin, ampicillin, SXT, and cephalothin, and higher sensitivity to imipenem, ciprofloxacin, nitrofurantoin, and ceftizoxime.

Table 1. : Frequency of Bacterial Agents Isolated From Urine Specimens and Their Relation to Sex in This Study

\begin{tabular}{llll}
\hline Isolated bacteria & No. $(\%)$ & Female, No, $(\%)$ & Male, No $(\%)$ \\
\hline Gram-Negative bacilli & & & \\
Escherichia coli & $152(66.08)$ & $136(89.5)$ & $16(10.5)$ \\
Klebsiella spp. & $22(9.6)$ & $18(81.8)$ & $4(18.2)$ \\
Proteus spp. & $4(1.70$ & $4(100)$ & $0(0)$ \\
Enterobacter spp. & $2(0.86)$ & $2(100)$ & $0(0)$ \\
Gram-Positive cocci & & & $4(15.4)$ \\
Staphylococcus saprophyticus & $26(11.3)$ & $22(84.6)$ & $0(0)$ \\
Staphylococcus aureus & $4(1.7)$ & $4(100)$ & $0(0)$ \\
Streptococcus group B & $10(8.69)$ & $10(100)$ & $2(20)$ \\
Enterococcus spp. & $10(8.69)$ & $8(80)$ & $26(11.3)$ \\
Total & $230(100)$ & $204(88.7)$ & \\
\hline
\end{tabular}


Klebsiella strains displayed a similar resistance pattern as for E. coli and showed high susceptibility to ciprofloxacin, ceftizoxime, nalidixic acid, and imipenem and high resistance to gentamicin, cephalothin, and ampicillin. On Proteus strains, ampicillin, nitrofurantoin, and SXT showed the least $(0 \%, 0 \%$, and $25 \%$, respectively) and ciprofloxacin, ceftizoxime, and imipenem showed the highest susceptibility $(100 \%, 100 \%$ and, $75 \%$, respectively) that were almost similar to the results with other Gram positive bacteria. The results were slightly different in Enterobacter as it can be seen the utmost resistance of Enterobacter was recorded to ampicillin and nalidixic acid while $100 \%$ susceptibility to other antibiotics was recorded. In this study, staphylococci were responsible for about $13 \%$ of UTIs cases; among these, S. saprophyticus isolates as most frequent Gram positive bacteria showed high resistance to ampicillin, tetracycline, and erythromycin, and high sensitivity to nitrofurantoin, gentamicin, norfloxacin, and vancomycin. The rates of resistance to 11 selected antimicrobial agents against Gram negative bacilli and to 9 selected antibiotics against Gram positive cocci are summarized in Table 2 and Table 3, respectively.

Table 2. Prevalence and Antimicrobial Resistance of Gram Negative Uropathogens Isolated in this Study

\begin{tabular}{|c|c|c|c|c|c|c|c|c|c|c|c|c|}
\hline Bacteria & No. & AM & $\mathbf{C P}$ & CT & IPM & GM & SXT & NA & CTX & FM & CAZ & CF \\
\hline E.coli & 152 & 73.69 & 26.32 & 15.79 & 15.79 & 73.69 & 69.74 & 60.53 & 38.16 & 9.22 & 55.27 & 88.16 \\
\hline $\begin{array}{l}\text { Klebsiella } \\
\text { spp. }\end{array}$ & 22 & 63.64 & 27.28 & 27.28 & 18.9 & 63.64 & 63.64 & 27.28 & 45.46 & 54.55 & 54.55 & 45.46 \\
\hline Proteus spp. & 4 & 100 & 0 & 0 & 25 & 50 & 75 & 50 & 50 & 100 & 50 & 25 \\
\hline $\begin{array}{l}\text { Enterobacter } \\
\text { spp. }\end{array}$ & 2 & 100 & $\mathbf{0}$ & $\mathbf{0}$ & $\mathbf{0}$ & 50 & $\mathbf{0}$ & 100 & $\mathbf{0}$ & $\mathbf{0}$ & $\mathbf{0}$ & 0 \\
\hline
\end{tabular}

Abbreviations: AM, Ampicillin; CP, Ciprofloxacin; CT, Ceftizoxime; IPM, Imipenem; GM, Gentamicin; SXT, Sulfamethoxazole-trimethoprim; NA, Nalidixic acid; CTX, Cefatoxime; FM, Nitrofurantoin; CAZ, Ceftazidime; CF, Cephalothin

Table 3. Prevalence and Antimicrobial Resistance of Gram Positive Uropathogens Isolated in this Study

\begin{tabular}{lllllllllll}
\hline Bacteria & No & AM & C & E & NOR & GM & V & FM & TE & SXT \\
\hline $\begin{array}{l}\text { Staphylococcus } \\
\text { saprophyticus }\end{array}$ & $\mathbf{2 6}$ & $\mathbf{9 2 . 3 1}$ & $\mathbf{6 1 . 5 4}$ & $\mathbf{9 2 . 3 1}$ & $\mathbf{3 0 . 7 7}$ & $\mathbf{3 0 . 7 7}$ & $\mathbf{7 . 7}$ & 7.7 & $\mathbf{9 2 . 3 1}$ & 77 \\
$\begin{array}{l}\text { Staphylococcus } \\
\text { aureus }\end{array}$ & $\mathbf{4}$ & $\mathbf{1 0 0}$ & $\mathbf{5 0}$ & $\mathbf{5 0}$ & $\mathbf{5 0}$ & $\mathbf{0}$ & $\mathbf{5 0}$ & $\mathbf{0}$ & $\mathbf{5 0}$ & $\mathbf{0}$ \\
$\begin{array}{l}\text { Streptococcus } \\
\text { group B }\end{array}$ & $\mathbf{1 0}$ & $\mathbf{3 0}$ & $\mathbf{2 0}$ & $\mathbf{3 0}$ & $\mathbf{4 0}$ & $\mathbf{7 0}$ & $\mathbf{8 0}$ & $\mathbf{0}$ & $\mathbf{7 0}$ & $\mathbf{8 0}$ \\
$\begin{array}{l}\text { Enterococcus } \\
\text { spp. }\end{array}$ & $\mathbf{1 0}$ & $\mathbf{4 0}$ & $\mathbf{4 0}$ & $\mathbf{7 0}$ & $\mathbf{1 0 0}$ & $\mathbf{1 0 0}$ & $\mathbf{0}$ & $\mathbf{0}$ & $\mathbf{1 0 0}$ & $\mathbf{1 0 0}$ \\
\hline
\end{tabular}

Abbreviations: AM, Ampicillin; C, Chloramphenicol; E, Erythromycin; NOR, Norfloxacin; GM, Gentamicin; V, Vancomycin; FM, Nitrofurantoin; TE, Tetracycline; SXT, Sulfamethoxazole-trimethoprim

\section{Discussion}

This study shows the distribution of microbial species isolated from patients with UTI at a diagnostic laboratory center in Warangal,India, and their susceptibility pattern to antimicrobial agents. Furthermore, we described the relationship between sex and isolated bacterial agents of UTI. Microbial infection of the UTI is one of the most common infectious diseases worldwide (19). Approximately 1 in 3 women will require antimicrobial treatment for a UTI before age 24 , and $40 \%$ to $50 \%$ of women will suffer from UTI during their lifetime (20). The sex distribution of patients in our study is consistent with those in other studies $(2,16,21)$ showing a statistically predominance of females $(88.69 \%$ of the positive cultures). It is assumed that the short urethra in girls predisposes them to ascending infection. Furthermore, management of micturition in women is important. Management mistakes made by women include cleaning perineum forward from the anus to the vulva (2) that can cause urinary tract infection.

Sexual activity has been reported to influence higher prevalence of UTI in females (22). Considering the fact that most of infecting organisms are commensals of perianal and vaginal regions, emphasis on personal hygiene especially in females may be important in reducing the incidence of UTI (18). Males are less prone to UTIs possibly because of their longer urethra and the presence of antimicrobial substances in prostatic fluid (23). Our study indicates that E. coli is still the most common cause of UTI in Warangal. This corresponds with the data obtained by other investigators $(3,15,17)$. In addition, coagulase negative Staphylococcus spp. was the most common cause of UTI among Gram positive bacteria. Recent studies have revealed the importance of coagulase negative Staphylococcus spp. in urinary tract infections (24).

Our findings demonstrated that these pathogens play an important role in UTI as $11.3 \%$ of cases in our study were affected by these bacteria. Klebsiella spp. with the rate of $9.6 \%$ was the third species that caused UTI. In Our study, as with previous studies, E. coli demonstrated a very high microbial resistance to antibiotics. 
The analyzed results of antibiotic susceptibility test showed that E. coli was highly resistant to gentamicin (73.69\%), ampicillin (73.69\%), SXT (69.74\%), and cephalothin (88.16\%). This is similar to previous studies in USA (25) and Iran (17). On the other hand, very low resistancewas detected to antibiotics such as ciprofloxacin (26.32\%), imipenem (15.79\%), nitrofurantoin (9.22\%), and ceftizoxime (15.79\%).

In the present study, the coagulase negative Staphylococcus showed $92.31 \%$ resistance to ampicillin, tetracycline, and erythromycin, respectively. Results showed that the best activity against these bacteria was achieved by nitrofurantoin, gentamicin, norfloxacin, and vancomycin that corresponds with other study in our region and other parts of the world $(26,27)$. Klebsiella spp. exhibited varying antibiotic resistance and showed higher resistance to gentamicin $(63.64 \%)$ and ceftazidime $(54.55 \%)$. Also Klebsiella spp. showed the lowest resistance to ciprofloxacin, ceftizoxime, and nalidixic acid (27.28\%). Surprisingly, in the present study, the susceptibility of Enterobacteriaceae to gentamicin was low while other studies in warangal reported high sensitivity to this antibiotic $(21,23)$ which can be due to uncontrolled administration of the drug.

A significant increase in resistance of pathogenic strains to SXT, ampicillin, and cephalothin has been found worldwide (28). On the other hand, the effective drugs for UTI are ciprofloxacin, nitrofurantoin, and imipenem in our region. Low resistance to these drugs was observed because they were not easily available and are relatively expensive compared to others. Thus, these drugs could be considered as alternative options in empirical treatment of UTIs (16). Ciprofloxacin as an option for remedy to UTIs has been considered since its multiple mechanisms of action seem to have enabled it to retain potent activity against E. coli. Ciprofloxacin shows high level of activity against E. coli in UTI compared to other commonly used agents such as ampicillin and SXT (29). The data suggest that these drugs should be used cautiously to ensure that resistance rates to these substantial antibiotics for UTIs do not increase.

In the last decades, the number of reports about appearance of bacteria with antibiotic resistance has increased all over the world (3). This study, like others, shows clearly that there are significant geographic differences in the susceptibility of commonly used antimicrobials against UTIs $(25,30)$. As a result, accurate knowledge on local epidemiology and patterns of antimicrobial resistance in uropathogens are essential to design a clinically effective therapy for UTI. However in the current study we observed a high level and generalized resistance to commonly used antibiotics particularly among Gram negative isolates. This rate of resistance, especially to Gentamicin is higher in our region than that observed in other countries such as Poland and Canada. Reducing the number of prescription for a particular antibiotic can lead to a decrease in resistance rates $(31,32)$. Studies like the present study are useful in determining local trends and risk factors for antimicrobial resistance and furthermore it is important to urge physicians and other health workers on performing antibiotic susceptibility test before blind antibiotic therapy.

With regard to continuous changing in causative bacteria isolated from patients with urinary tract infection and antibiotic sensitivity pattern, we recommend annual determination of bacterial sensitivity in populations as a guideline to be respected by physicians. Furthermore, considering susceptibility pattern of antibiotic agents for UTI therapy, particularly caused by E. coli as the most significant uropathogen, it seems that the drugs like ampicillin and SXT should be prescribed cautiouslyespecially against to those, demonstrate appropriate respond to other antibiotics such as ciprofloxacin and nitrofurantoin.

\section{References}

[1]. Hooton TM. A simplified approach to urinary tract infection. Hosp Pract (Off Ed). 1995;30(2):23-30.

[2]. Modarres s, Nassiri Oskoii N. Bacterial etiologic agents of urinary tract infection in children in the Islamic Republic of Iran. East Mediterran Health J. 1997;3(2):290-5.

[3]. Ranjbar R, Haghi-Ashtiani M, Jafari NJ, Abedini M. The prevalence and antimicrobial susceptibility of bacterial uropathogens isolated from pediatric patients. Iranian J Pub Health. 2009;38(2).

[4]. Vasquez Y, Hand W. Antibiotic susceptibility patterns of community-acquired urinary tract infection isolates from female patients on the US (Texas)-Mexico border. J Appl Res. 2004;4(2):321-6.

[5]. Behrman R, Kliegman R, Jenson H. Nelson Textbook of Pediatrics. Philadelphia: WB Saunders Company. Lippincott Williams \& Wilkins; 1989.

[6]. Feld LG, Mattoo TK. Urinary Tract Infections and Vesicoureteral Reflux in Infants and Children. Pediatr Rev. 2010;31(11):451-63.

[7]. Inabo H, Obanibi H. Antimicrobial susceptibility of some urinary tract clinical isolates to commonly used antibiotics. Afr J Biotechnol. 2006;5(5):487-9.

[8]. Al Sweih N, Jamal W, Rotimi VO. Spectrum and antibiotic resistance of uropathogens isolated from hospital and community patients with urinary tract infections in two large hospitals in Kuwait. Med Princ Pract. 2005;14(6):401-7.

[9]. Kolawole A, Kolawole O, Kandaki-Olukemi Y, Babatunde S, Durowade K, Kolawole C. Prevalence of urinary tract infections (UTI) among patients attending Dalhatu Araf Specialist Hospital, Lafia, Nasarawa State, Nigeria. Int J Medicinal Med Sci. 2009;1(5):163-7.

[10]. Mandell GL, Bennett JE, Dolin R. Principles and practice of infectious diseases. Churchill Livingstone. 2005:881-2.

[11]. Hvidberg H, Struve C, Krogfelt KA, Christensen N, Rasmussen SN, Frimodt-Moller N. Development of a long-term ascending urinary tract infection mouse model for antibiotic treatment studies. Antimicrob Agents Chemother. 2000;44(1):156-63.

[12]. Ramesh N, Sumathi CS, Kannan VR. Urinary Track Infection and Antimicrobial Susceptibility Pattern of Extended Spectrum of Beta Lactamase Producing Clinical Isolates. Adv Biol Res. 2008;2(5-6):78-82.

[13]. Jellheden B, Norrby RS, Sandberg T. Symptomatic urinary tract infection in women in primary health care. Bacteriological, clinical and diagnostic aspects in relation to host response to infection. Scand J Prim Health Care. 1996;14(2):122-8. 
[14]. Ronald A. The etiology of urinary tract infection: traditional and emerging pathogens. Am J Med. 2002;113 Suppl 1A:14S-9S.

[15]. Hryniewicz K, Szczypa K, Sulikowska A, Jankowski K, Betlejewska K, Hryniewicz W. Antibiotic susceptibility of bacterial strains isolated from urinary tract infections in Poland. J Antimicrob Chemother. 2001;47(6):773-80.

[16]. Beyene G, Tsegaye W. Bacterial uropathogens in urinary tract infection and antibiotic susceptibility pattern in jimma university specialized hospital, southwest ethiopia. Ethiop J Health Sci. 2011;21(2):141-6.

[17]. Amin M, Mehdinejad M, Pourdangchi Z. Study of bacteria isolated from urinary tract infections and determination of their susceptibility to antibiotics. Jundishapur J Microbiol. 2011;2(3):118-23. 